Revue

Revue de l'histoire des religions

de Ihistoire des religions

3 | 2018

Varia

\title{
Alain TESTART, Art et religion de Chauvet à Lascaux
}

Paris, Gallimard, « Bibliothèque illustrée des Histoires », 2016

\section{Christophe Lemardelé}

\section{OpenEdition}

\section{Journals}

Édition électronique

URL : http://journals.openedition.org/rhr/8985

DOI : $10.4000 /$ rhr.8985

ISSN : 2105-2573

Éditeur

Armand Colin

Édition imprimée

Date de publication : 1 septembre 2018

Pagination : $531-533$

ISBN : 978-2-200-93188-9

ISSN : 0035-1423

Référence électronique

Christophe Lemardelé, "Alain TESTART, Art et religion de Chauvet à Lascaux », Revue de l'histoire des religions [En ligne], 3 | 2018, mis en ligne le 01 septembre 2018, consulté le 14 janvier 2021. URL : http://journals.openedition.org/rhr/8985; DOI : https://doi.org/10.4000/rhr.8985

Ce document a été généré automatiquement le 14 janvier 2021.

Tous droits réservés 


\title{
Alain TESTART, Art et religion de Chauvet à Lascaux
}

Paris, Gallimard, « Bibliothèque illustrée des Histoires », 2016

\author{
Christophe Lemardelé
}

\section{RÉFÉRENCE}

Alain TESTART, Art et religion de Chauvet à Lascaux, Paris, Gallimard, « Bibliothèque illustrée des Histoires », 2016, 373 p., 21,5 cm, 26 €, ISBN 978-2-07-269364-9.

1 L'édition de cet ouvrage posthume a été établie par Valérie Lécrivain, qui soutint une thèse sous la direction d'Alain Testart et qui est sa légataire scientifique. Dans l'avantpropos, elle rappelle que le livre, aboutissement d'un travail difficile et remarquable, requérant une iconographie considérable, est le fruit d'un séminaire que tint l'anthropologue dans les années 2006-2007. Directeur de recherche émérite au CNRS, il faisait toutefois cours devant fidèles et préhistoriens de manière à mettre à l'épreuve ses réflexions. C'est donc à une analyse minutieuse de l'art pariétal du Paléolithique supérieur que se livre Alain Testart dans ce très bel ouvrage, analyse prolongée par des appendices concernant les Aborigènes d'Australie puisque sa thèse est d'identifier cet art comme étant une vision totémique du monde.

Dans l'introduction, l'A. énonce son projet qui se veut humble et dans les pas du célèbre archéologue André Leroi-Gourhan. Il ne s'agit en effet pas pour lui de plaquer un savoir ethnographique sur des représentations préhistoriques qui garderont, quoi qu'il en soit, leur part de mystère, sans toutefois s'abstenir de mobiliser de telles connaissances extérieures. La problématique est énoncée au travers d'une réflexion faussement sérieuse sur l'art roman, gothique et baroque, la question n'étant plus : « connaissant la religion, qu'en est-il de son expression artistique? mais connaissant l'expression artistique, que dire de la religion qu'elle est censée traduire?» (p.23). Toute la difficulté concernant les représentations religieuses préhistoriques réside en effet dans 
le fait que l'art peut en être une manifestation. Or, comment l'interpréter et comment faire la part du religieux et du symbolique?

3 L'ouvrage est organisé en trois parties : 1/ Analyse iconographique ; 2/ Signes ; 3/ Organisation spatiale. Avec un plaisir non dissimulé, l'A.s'applique à analyser l'iconographie préhistorique dans de courts chapitres, somptueusement illustrés, qui proposent souvent en fin d'analyse un résumé et une ou des «implications », c'est-àdire une ou des conclusions à tirer. L'une de ces conclusions consiste d'abord à établir que l'art pariétal n'est pas narratif. Il est certes réaliste mais pratique un naturalisme limité : l'animal est sans lien avec son milieu naturel, les différentes espèces ne s'affrontent pas entre elles, seuls les animaux d'une même espèce sont en interaction. Cet art donne donc à voir d'abord et avant tout une classification selon les espèces, il est "l'expression d'une pensée classificatoire» (p. 79). Deuxième conclusion d'importance : l'homme n'est pas représenté en interaction avec les animaux et il ne l'est que sous une forme inachevée dans les grottes, notamment sous la forme d'êtres mi-hommes, mi-animaux (thérianthropes), alors que la représentation humaine trouve son plein achèvement, à l'extérieur des cavernes, dans l'art mobilier. Cette humanité embryonnaire implique dès lors que la grotte est conçue comme un état mythique, une origine du monde (p. 106-107). La seconde implication est tout aussi importante car cela suppose encore que les espèces représentées ne le soient pas dans un contexte d'art animalier mais bien de représentation du monde tel que les hommes pouvaient le penser. Or, d'un point de vue anthropologique, seule la pensée totémique peut conduire à classer des espèces animales pour, en fait, y ranger les hommes dans des catégories sociales (p. 108-109). Ici, l'animal est une métaphore pour l'homme: les différentes espèces animales traduisent les différences entre les hommes selon leur clan. Pour l'A., quoi qu'il en soit, il ne s'agit pas de représentations chamaniques tant la pensée classificatoire à l'œuvre dans les grottes est distincte d'une relation aux espritsanimaux impliquant la chasse.

4 Les grottes ne recèlent pas que des images animalières, elles comportent également des signes plus abstraits, bien difficiles à interpréter, et d'autres sans équivoque, notamment des vulves en nombre - en fait, des triangles avec un trait vertical schématisant le sexe féminin - et quelques phallus humains. Pour Alain Testart, les représentations sexuelles féminines sont omniprésentes dans la grotte par le biais des signes. À ce titre, et en véritable historien de l'art, il fait un détour par les fameuses statuettes de « Vénus » préhistoriques à partir desquelles et de leur parfaite symétrie il déduit quelques canons esthétiques lui permettant de proposer une nouvelle théorie des signes. Vulve, triangle, tectiforme, claviforme, etc., sont des images métonymiques ou des représentations schématiques de la femme. Alain Testart conclut: "que les hommes du Paléolithique aient apposé tellement de signes de la féminité sur les images d'animaux, le plus souvent au fond de grottes difficiles d'accès, est la preuve que la reproduction était une préoccupation centrale de leur religion» (p. 231). Tandis que dans les religions à chamans, de manière générale, la religion est déterminée par la survie et donc la question de la chasse, le gibier étant bien souvent retenu par une entité spirituelle (guardian spirit), dans les grottes, un peu à l'instar des sites sacrés des Aborigènes d'Australie, il semblerait que la religion se concentre sur la perpétuation de la vie, les espèces animales étant "préformées ", en latence (p. 125-129) - la différence (soulignée par V. Lécrivain) provenant du fait que les cavernes regorgent d'animaux tandis que les sites aborigènes regorgent $d$ '« esprits-enfants » (p. 294). Dès lors, l'ultime partie de l'ouvrage concernant l'organisation spatiale des grottes devient passionnante, 
les animaux représentés entrant en mouvement circulaire, tout en venant ponctuer la démonstration.

Dans la conclusion de l'ouvrage, «La grotte est femme », rédigée par Valérie Lécrivain, trois lignes de force sont mises en exergue : 1/ l'art pariétal obéit à une vision du monde structurée par les différences entre les espèces animales; 2/ la grotte représente les temps mythiques des origines d'où tout est engendré et où les hommes sont encore inachevés; $3 /$ les hommes sont associés aux espèces animales dans une relation totémique ancrée dans une représentation cosmogonique (p. 297-305). Les appendices rédigés par Alain Testart lui-même prolongent cette thèse en développant les conceptions totémiques des autochtones australiens - c'est en soi un petit manuel sur la religion aborigène (p. 311-331). Le dernier appendice, intitulé « Les deux types de religions chez les chasseurs-cueilleurs ", est particulièrement intéressant car il permet de confronter religion totémique, sans entités surnaturelles présentes comme chez les Aborigènes, et religions « chamaniques ». Or, si c'est bien dans cette dichotomie que se situe l'enjeu des grottes du Paléolithique, la perspective peut être élargie à l'évolution des conceptions religieuses.

Alain Testart a raison de distinguer de véritables religions chamaniques, en Sibérie et en Amazonie, de religions avec un guardian spirit chez les Inuit et, plus largement, en Amérique du Nord (p. 258-259); il reste que toutes ont un chaman en mesure d'agir sur des entités surnaturelles, certes avec un pouvoir moindre pour le chaman des secondes. Il ne faudrait pas retomber dans les ornières de l'évolutionnisme d'antan, mais une catégorisation des espèces animales sans que celles-ci soient liées à de quelconques esprits nécessitant un chaman semble antérieure à ces conceptions, ce qu'attesterait l'interprétation proposée dans l'ouvrage par l'anthropologue pour des conceptions du Paléolithique. Or, des populations situées dans des périphéries continentales comme les San du Kalahari, avec une conception «chamanique » encore embryonnaire (p. 331), ou, surtout, comme les Selk'nam de la Terre de Feu, ayant « un maître du gibier (des guanacos) et une initiation très stricte comparable à celle de l'Australie » (p. 347, n. 21), pourraient nous conduire à penser qu'il n'importe pas seulement de classer ces conceptions religieuses mais de les appréhender sous l'angle de l'évolution des conceptions religieuses. Le fait que des religions à chamans forment un continuum de l'Asie septentrionale à l'Amérique atteste sans nul doute la diffusion de ces conceptions accompagnant des populations en mouvement. La religion australienne, quelque peu isolée dans son « bout du monde », serait donc bien dépositaire d'une forme de religion très ancienne.

\section{AUTEURS}

\section{CHRISTOPHE LEMARDELÉ}

Laboratoire Orient \& Méditerranée, UMR 8167. 